\title{
Rezidivschutz: Besser ASS als gar nichts
}

Für VTE-Patienten, die nicht längerfristig antikoaguliert werden können, ist ASS eine Alternative.

ASS schützt nach venöser Thromboembolie (VTE) lange nicht so wirksam vor einem Rezidiv wie eine orale Antikoagulation (Risikoreduktion $>80 \%$ ), ist aber besser als gar keine Prophylaxe.

Das belegt die INSPIRE-Studie, in der Patienten mit idiopathischer VTE und beendeter Warfarintherapie mit $100 \mathrm{mg} / \mathrm{d}$ ASS oder Placebo weiterbehandelt wurden. Innerhalb von vier Jahren kam es unter ASS jährlich bei 5,1\% und unter Placebo bei 7,5\% zu einem Rezidiv. Das entspricht einer relativen Risikoreduktion von $32 \%$ durch den Plättchen- hemmer. Um eine VTE zu verhindern, müssen demnach 42 Patienten behandelt werden.

\section{TVT und Embolien gleichermaßen reduziert}

Tiefe Venenthrombosen und Lungenembolien wurden in gleichem Maße reduziert. Die absolute Risikominderung war im ersten Jahr am größten (5,4 vs. 11,0\%).

Blutungen traten nicht häufiger auf als mit Placebo (1,1 vs. $0,7 \%$ pro Jahr). Bei Patienten, die etwa wegen Kontraindikationen nicht antikoaguliert werden können, sollte daher die ASS-Gabe geprüft werden.

Circulation 2014; 130: 1062-1071
... fördern alle ora-

Ien Kontrazeptiva,

die Entstehung von

Thrombosen. Die in Präpa-

raten der dritten Generation

enthaltenen Gestagene Gestoden,

Drospirenon, Cyproteron und Desogestrel sind mit einem höheren Risiko belastet als Levonorgestrel.

BMJ 2015; 350: h2135

... kommt es nach einer Hüftfraktur nur im ipsilateralen Bein zu einem signifikanten Anstieg des Thromboserisikos.

BMJ Open 2015; 5:e006956

... erleiden Patienten, die nicht steroidale Antirheumatika (NSAR) einnehmen, häufiger venöse Thromboembolien. Einer Metaanalyse zufolge ist ihr Risiko gegenüber Nichtanwendern um 80\% erhöht.

Rheumatology 2015; 54: 736-742

\section{Eine verzögerte Diagnose kann katastrophale Folgen haben: in Form einer Lungenembolie oder eines postthrombotischen Syndroms}

- thrombosen (TVT) und Lungenembolie auch DOAK eingesetzt werden: Sowohl die direkten Faktor-Xa-Hemmer Apixaban (Eliquis ${ }^{\circledR}$ ) und Rivaroxaban (Xarelto ${ }^{\star}$ ) als auch der direkte Thrombinhemmer Dabigatran (Pradaxa ${ }^{\oplus}$ ) sind in dieser Indikation zugelassen.

In Studien waren sie der Standardtherapie aus NMH und Vitamin-K-Antagonist in der Wirksamkeit ebenbürtig, führten aber seltener zu Blutungen. Das gilt ebenso für Edoxaban (Lixiana ${ }^{\circledR}$ ); der selektive Faktor-Xa-Hemmer hat vor Kurzem die EU-Zulassung zur Therapie und Rezidivprophylaxe der TVT und Lungenembolie erhalten. Die Marktzulassung basiere auf den Ergebnissen zweier Phase-III-Studien, teilt das Unternehmen Daiichi Sankyo mit. Mit Dabigatran wird die TVT-Therapie wie gewohnt mit einem NMH eingeleitet und nach fünf Tagen auf den oralen Wirkstoff umgestellt. Mit Apixaban und
Rivaroxaban kann die Antikoagulation von Anfang an oral erfolgen, ohne NMHTherapie. Auf eine ausreichende Nierenfunktion ist auch bei den DOAK zu achten. Im Gegensatz zur Behandlung mit Vitamin-K-Antagonisten sind keine Gerinnungskontrollen erforderlich.

Ein Manko gegenüber den Kumarinderivaten ist, dass noch keine spezifischen Antidots verfügbar sind. An der Entwicklung solcher Gegenmittel wird jedoch intensiv gearbeitet; für Idarucizumab als Dabigatran-Antidot wurde bereits die Zulassung beantragt.

In der aktualisierten Leitlinie zu Diagnostik und Therapie der Venenthrombose und der Lungenembolie werden erstmals die neuen direkten oralen Antikoagulanzien berücksichtigt sein. Die Leitlinie wird in Kürze erwartet.

(Beate Schumacher)

[1] MMW-Fortschr Med 2014; SH 2: 59-63

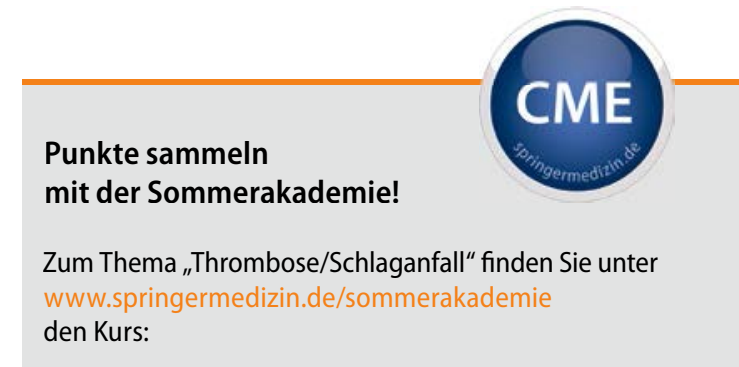

"Oft andere Ursachen als bei älteren Patienten: Schlaganfall bei jungen Menschen"

Mit e.Med teilnehmen

Mit e.Med haben Sie nicht nur Zugriff auf die aktuelle Sommerakademie, Sie können das komplette Angebot von Springer Medizin nutzen - zusätzlich erhalten Sie eine gedruckte Fachzeitschrift Ihrer Wahl. Mit rund 1600 Fortbildungskursen, davon über 500 CME-zertifiziert, ist die e.Akademie Deutschlands erste Adresse für Online-CME-Fortbildung.

Nutzen Sie die Sommerzeit, um reichlich CME-Punkte zu sammeln!

30 Tage kostenlos

Unser Kundenservice steht Ihnen wochentags (10-17 Uhr) kostenlos zur Verfügung: Tel. 08007780777 kundenservice@springermedizin.de 